ENCYCLOPÉDIE Encyclopédie berbère

BERBERE

17| 1996

17 | Douiret - Eropaei

\title{
Émigration
}

(kabyle)

\section{K. Direche-Slimani}

\section{CpenEdition}

Journals

Édition électronique

URL : http://journals.openedition.org/encyclopedieberbere/2140

DOI : 10.4000/encyclopedieberbere. 2140

ISSN : 2262-7197

\section{Éditeur}

Peeters Publishers

\section{Édition imprimée}

Date de publication : 1 août 1996

Pagination : 2614-2617

ISBN : 2-85744-872-4

ISSN : 1015-7344

Référence électronique

K. Direche-Slimani, «Émigration », Encyclopédie berbère [En ligne], 17| 1996, document E16, mis en ligne le 01 juin 2011, consulté le 24 septembre 2020. URL : http://journals.openedition.org/ encyclopedieberbere/2140; DOI : https://doi.org/10.4000/encyclopedieberbere.2140

Ce document a été généré automatiquement le 24 septembre 2020.

(c) Tous droits réservés 


\section{Émigration}

\section{(kabyle)}

\section{K. Direche-Slimani}

1 L'importance et la régularité de la main-d'œuvre kabyle en France et dans le temps est indéniable et de nombreuses traces dans les archives et documents coloniaux (notamment dans les nombreux rapports administratifs consacrés aux mouvements migratoires des travailleurs coloniaux) le prouvent. Avant la première guerre mondiale, l'émigration vers la Métropole était constituée de ce que l'on appelait à l'époque les convoyeurs kabyles; il existait entre la France et l'Algérie un va et vient continuel de bateaux qui opéraient des transferts massifs de bovins et de chevaux. Pendant longtemps la Kabylie fut la principale pourvoyeuse de cette main-d'œuvre ; un rapport d'une commission d'enquête estime en 1912 près de 10000 kabyles travaillant en France particulièrement dans les Bouches-du-Nord et dans le Pas-de-Calais. En 1934, elle fournissait les $3 / 4$ des émigrés et en 1950, 50 à $60 \%$ des travailleurs partis en France étaient d'origine kabyle. C'est dire à quel point l'émigration est une donnée sociologique et économique fondamentale de la Kabylie et la manifestation la plus évidente de son sous-développement économique et du déséquilibre des structures traditionnelles et rurales (la paysannerie étant la principale pourvoyeuse de maind'œuvre).

2 L'indépendance de l'Algérie n'a pas entraîné de profonds bouleversements et n'a surtout pas arrêté l'émigration vers la France (l'influence coloniale donnant encore une préférence d'émigration aux anciennes colonies). Mais la part de la Kabylie n'est plus aussi importante; l'émigration s'étant étendue à d'autres régions de l'Algérie, notamment aux zones arabophones : l'Oranie et les Hauts-Plateaux constantinois.

3 Tenter de faire l'histoire de l'émigration kabyle, la replacer dans l'histoire des mouvements migratoires algériens vers la France et proposer des statistiques n'est pas une entreprise aisée. En effet, les spécificités culturelles et identitaires de l'immigration n'ont jamais été perçues en tant que telles par les chercheurs qui ont mit l'accent sur l'uniformité des masses migratoires et tenant compte uniquement du critère de distinction qu'est la nationalité d'origine; le paramètre linguistique n'entrant pas en considération. 
4 En règle générale, l'émigration d'origine berbère a toujours été confondue dans l'émigration dite arabe et il est souvent difficile de distinguer, dans les documents qui existent, la dimension kabyle des migrations vers la France.

On peut avancer aujourd'hui quelques chiffres (voir tableau) qui prouvent la dimension phénoménale qu'a pu connaître l'émigration kabyle qui a vidé parfois certains villages et à des périodes bien précises des trois quarts de sa population masculine et a marqué la Kabylie psychologiquement et matériellement.

6 Historiquement, c'est l'insurrection de 1871 et le phénomène de dépossession des terres lancé par la colonisation qui avaient déjà ébauché le premier mouvement migratoire des Kabyles vers la Tunisie, puis vers la France. La politique coloniale agraire et l'écroulement des unités traditionnelles comme la tribu kabyle avait amorcé les premiers départs massifs. Mais cet exil d'origine politique reste somme toute marginal et assez exceptionnel. Ce sont des facteurs démographiques et économiques qui vont être à l'origine de ce processus migratoire.

7 La surpopulation d'une terre extrêmement morcelée, pauvre vivant d'une agriculture et arboriculture de montagne, l'absence en Kabylie de grandes villes et de pôles urbains importants pouvant drainer une main-d'œuvre nombreuse et créer des emplois expliquent l'importance des départs vers les grandes villes d'Algérie (notamment Alger) avec l'installation de familles entières puis vers la France. L'apprentissage du salariat va contribuer à rendre les départs de plus en plus fréquents et à présenter l'émigration comme la source de revenus la plus substantielle et la plus rentable pour la Kabylie. La prolétarisation des paysans kabyle activera l'abandon d'un certain nombre de traditions agraires ; un mouvement d'abandon des terres consécutif à un fort exode rural accentuera la rupture totale avec le système productif traditionnel (Lacoste, 1984).

8 Cette émigration longtemps temporaire, composée exclusivement d'hommes, forte d'une longue expérience du voyage va s'installer plus longuement en France et s'ancrera davantage avec les regroupements familiaux. Mais elle ne se contentera pas de développer uniquement des relations de travail ; elle sera un lieu intense d'action politique pour tous les déçus de la Révolution algérienne et pour tous les opposants au gouvernement algérien, un terrain très actif pour les groupes politiques berbéristes. L'existence de ces groupes a été doublement occultée dans l'histoire nationale algérienne et dans l'histoire de l'immigration algérienne en France. En effet les revendications culturelles et l'affirmation d'une appartenance identitaire berbère ont été considérées comme une fausse note dans le crédo arabo-islamique professé par les partis nationalistes algériens (ENA, PPA-MTLD, FLN). Le discours idéologique des berbéristes est double; il est à la fois politique, avec la revendication d'une identité berbère et la place effective qu'elle doit tenir dans les institutions algériennes, et longtemps culturel, avec tout le travail de valorisation culturelle et intellectuelle pour sortir du cadre folklorisant et muséographique dans lequel la culture berbère a été maintenue. 
Localisation en France des groupes d'émigrés algériens (supérieurs à 100) en juin 1950

\begin{tabular}{|c|c|c|}
\hline Départements & $\begin{array}{c}\text { Nombre total } \\
\text { d'émigrés algériens }\end{array}$ & $\begin{array}{c}\text { Nombre d'émigrés de } \\
\text { Grande et Petite Kabylie }\end{array}$ \\
\hline Seine & 12062 & 9349 \\
\hline Bouches-du-Rhône & 2292 & 2292 \\
\hline Moselle & 1587 & 1422 \\
\hline Rhône & 1299 & 1299 \\
\hline Nord & 2304 & 1295 \\
\hline Gard & 956 & 835 \\
\hline Loire & 764 & 764 \\
\hline Meurthe-et-Moselle & 477 & 477 \\
\hline Seine-Maritime & 399 & 399 \\
\hline Ardennes & 160 & 160 \\
\hline Ain & 130 & 130 \\
\hline Isère & 234 & 126 \\
\hline Oise & 125 & 125 \\
\hline Seine-et-Oise & 120 & 120 \\
\hline Savoie & 102 & 102 \\
\hline
\end{tabular}

9 L'émigration a réellement été un terrain privilégié d'expression identitaire, un lieu de travail culturel intense et de combat politique actif. Celui-ci a certes été mené par des minorités très politisées, qui ne sont pas représentatives d'une émigration de travail ; mais elles ont impulsé une réelle dynamique identitaire et politique et une motivation certaine pour la recherche dans le champ d'études berbères particulièrement en ce qui concerne les travaux de linguistique. La politique d'uniformisation culturelle et linguistique de l'Algérie indépendante ont fait de l'émigration kabyle le lieu d'opposition politique le plus actif et le plus productif en matière d'édition, de réalisations universitaires, pédagogiques (apprentissage de la langue berbère avec l'élaboration de manuels pédagogiques), musicales, de diffusion d'idées, de développement $\mathrm{du}$ réseau associatif. L'émigration kabyle est devenue la caisse de résonance (Chaker, 1989) du discours politique algérien. Elle a permis à une opposition contestataire de s'exprimer: dans un premier temps avec l'Académie Berbère, principalement, qui a opéré un véritable travail de défrichement en matière linguistique notamment avec la réutilisation du vieil alphabet berbère sous la forme du Tifinagh; dans un deuxième temps, avec le Groupe d'Études Berbères de Vincennes et les Ateliers Imedyazen qui ont entrepris une démarche de crédibilisation à la fois scientifique et politique dans une optique plus culturaliste. Ces groupes politisés (dont une partie des membres militent aujourd'hui au sein du FFS ou du RCD) ont fait de l'émigration un lieu où la conscience identitaire était la plus aiguë jusqu'à ce que le Printemps Berbère de 1980 ait amorcé en Algérie même le processus politico-culturel que l'on connaît.

10 Aujourd'hui, l'immigration kabyle reste un lieu privilégié de diffusion d'idées (bon nombre de réalisations musicales, littéraires ou scientifiques sont encore effectuées en France). Elle est depuis quelques années, le centre d'une mobilisation très active autour des questions de la langue kabyle et de son apprentissage. Un phénomène de réemergeance identitaire se cristallise autour de la défense de la langue et se développe auprès des jeunes issus de l'émigration. Dans cette quête des origines, c'est la 
réappropriation d'un ensemble de repères classiques au monde kabyle puis berbère qui font partie de tout un patrimoine symbolique. Cette jeunesse dont une partie milite au sein de certaines associations (Associations des Juristes Berbères, Association des Étudiants Berbères...), tient un discours très valorisant sur sa culture d'origine tout en prônant une totale adéquation aux valeurs de la Troisième République. Comme si en se réappropriant son origine, on se donnait les moyens de mieux réussir son intégration.

11 L'émigration kabyle a, tout au long du $\mathrm{xx}^{\mathrm{e}}$ siècle, maintenu une constance remarquable dans l'affirmation de sa conscience identitaire. Elle ne s'est pas limitée à la présence d'un homo economicus kabyle; elle a parallèlement développé et maintenu une tradition ancienne de promotion et de sauvegarde du patrimoine berbère. Espace de travail et d'exil, passerelle entre deux mondes, deux sociétés, deux cultures, lieu de combat et d'affirmation politique, l'émigration kabyle n'a jamais rompu ses liens avec le pays d'origine même si parfois une nostalgie douloureuse et exacerbée se constate avec le phénomène de réappropriation culturelle et identitaire des générations nouvelles.

\section{BIBLIOGRAPHIE}

CHAKER S., « Berbérité et émigration kabyle », Peuples Méditerranéens, n 31-32, 1985.

CHAKER S., Berbères aujourd'hui, Edisud, Aix-en-Provence, 1989.

DIRECHE-SLIMANI K., Histoire de l'émigration kabyle en France au $\mathrm{XX}^{\mathrm{e}}$ siècle : réalités culturelles et réappropriations identitaires, Doctorat Nouveau Régime d'Histoire, Aix-en-Provence, 1992.

TEMIME E., SAYD A., JORDI J.-J., Le choc de la décolonisation (1945-1990), Histoire des migrations à Marseille, tome IV, Aix-en-Provence, Edisud, 1991.

\section{INDEX}

Mots-clés : Algérie, Emigration, Ethnologie, Géographie, Kabyle 